\title{
Semitendinosus Tendon for Solitary Use in Anterior Cruciate Ligament Reconstruction
}

\author{
Brian Bundi, James Kigera, Gichambira Gikenye \\ School of Medicine, University of Nairobi
}

Correspondence to: Mr. Brain Bundi, P.O Box 19796-00202, Nairobi. Email: nyamweya9083@gmail.com

\begin{abstract}
Background: The use of a combined graft of both semitendinosus (ST) and gracilis (G) tendons in anterior cruciate ligament (ACL) reconstruction may cause weakness in knee flexion. It has since been proposed that ST be used alone since sparing G leads to near complete preservation of flexion strength. The use of the semitendinosus tendon as a solitary graft for reconstruction of the anterior cruciate ligament requires adequate tendon length $(>28 \mathrm{~cm})$ and four strand construct diameter $(>8 \mathrm{~mm})$. This study sought to determine the dimensions of the semitendinosus tendon graft among Kenyans. Methods: Forty pairs of ST tendons were harvested from formalin fixed cadavers by use of a tendon stripper. Their lengths were measured after which they were folded into four strand constructs whose diameter was obtained by sizing tunnels. Descriptive statistics and analysis was done using SPSS version 21.0. Results: The average ST tendon length was $29.80 \pm 3.59 \mathrm{~cm}$ and $67.5 \%$ of all
\end{abstract}

\section{Introduction}

A torn anterior cruciate ligament (ACL) results in knee instability. Tendon allografts and autografts have been used successfully to reconstruct a torn ACL. However, allografts have been associated with a higher rate of failure, especially in the young and active, leading to a preference for autografts (1). Autografts include the patella, semitendinosus and gracilis tendons. Even though autografts generally have good outcomes, donor site morbidity remains a significant complication of patella tendon autografts $(2,3)$. The semi tendinosus and gracilis tendon grafts are, therefore, becoming a common choice in ACL reconstruction (4-7). Reconstructing the ACL using a combined tendon graft from the semitendinosus (ST) and gracilis $(\mathrm{G})$ tendons restores knee function and stability $(6,8,9)$. However, harvesting both ST and G tendons may result in strength deficits in knee flexion and internal rotation $(2,10,11)$. With both muscles tendons had a length $\geq 28.0 \mathrm{~cm}$. The mean four strand construct diameter was $7.89 \pm 0.61 \mathrm{~mm}$ and $56 \%$ of all tendons had a thickness $\geq 8.00 \mathrm{~mm}$. Considering tendon adequacy to be the presence of both sufficient ST tendon length and four strand construct thickness, $51 \%$ of all tendons were adequate for solitary use. Conclusion: The use of ST as a solitary graft in ACL reconstruction may be feasible among Kenyans as a good proportion of our sample had adequate dimensions. We suggest that the ST tendon be harvested first during reconstruction as it may be sufficient by itself hence no need to harvest gracilis tendon.

Keywords: ACL Reconstruction, Semitendinosus Tendinosus, Solitary Use, Adequacy

Ann Afr Surg. 2016;13(2): 59-62.

DOI: http://dx.doi.org/10.4314/aas.v13i2.6

being knee flexors, sparing one of them may minimize the strength deficits.

The use of the ST tendon as a solitary tendon in ACL reconstruction has been proposed since this tendon is thicker than the $\mathrm{G}$ tendon and there is near complete preservation of knee flexion strength when $G$ is spared $(12,13)$. However, a solitary ST tendon must be at least $28 \mathrm{~cm}$ long and, upon folding to a four strand construct, have a minimum diameter of $7 \mathrm{~mm}$ for use in ACL reconstruction (9). There are reports of higher rates of failure in constructs less than $8 \mathrm{~mm}$ (14). Some authors have subsequently suggested a minimum sufficient diameter of $8 \mathrm{~mm}$ (15). Data from studies done in other populations suggests that using a solitary ST may not always be feasible as the tendon graft dimensions may be inadequate for fixation (1618). This study therefore, sought to determine the dimensions of the ST tendon among Kenyans. 


\section{Methods}

Forty formalin fixed cadavers without gross damage to the lower limbs or scars to the posterior thigh and medial knee regions were included in the study. An incision was made along the whole length of the posterior thigh towards the medial knee. Blunt dissection was used to expose the ST muscle and tendon. The ST tendon was harvested by use of a tendon stripper and its length obtained by tape measurement. Each tendon was then folded into a four strand construct of equal strand length and the thickness of the four strand construct obtained by use of sizing tunnels (Figure 1 and 2). Data were recorded and descriptive statistics obtained.
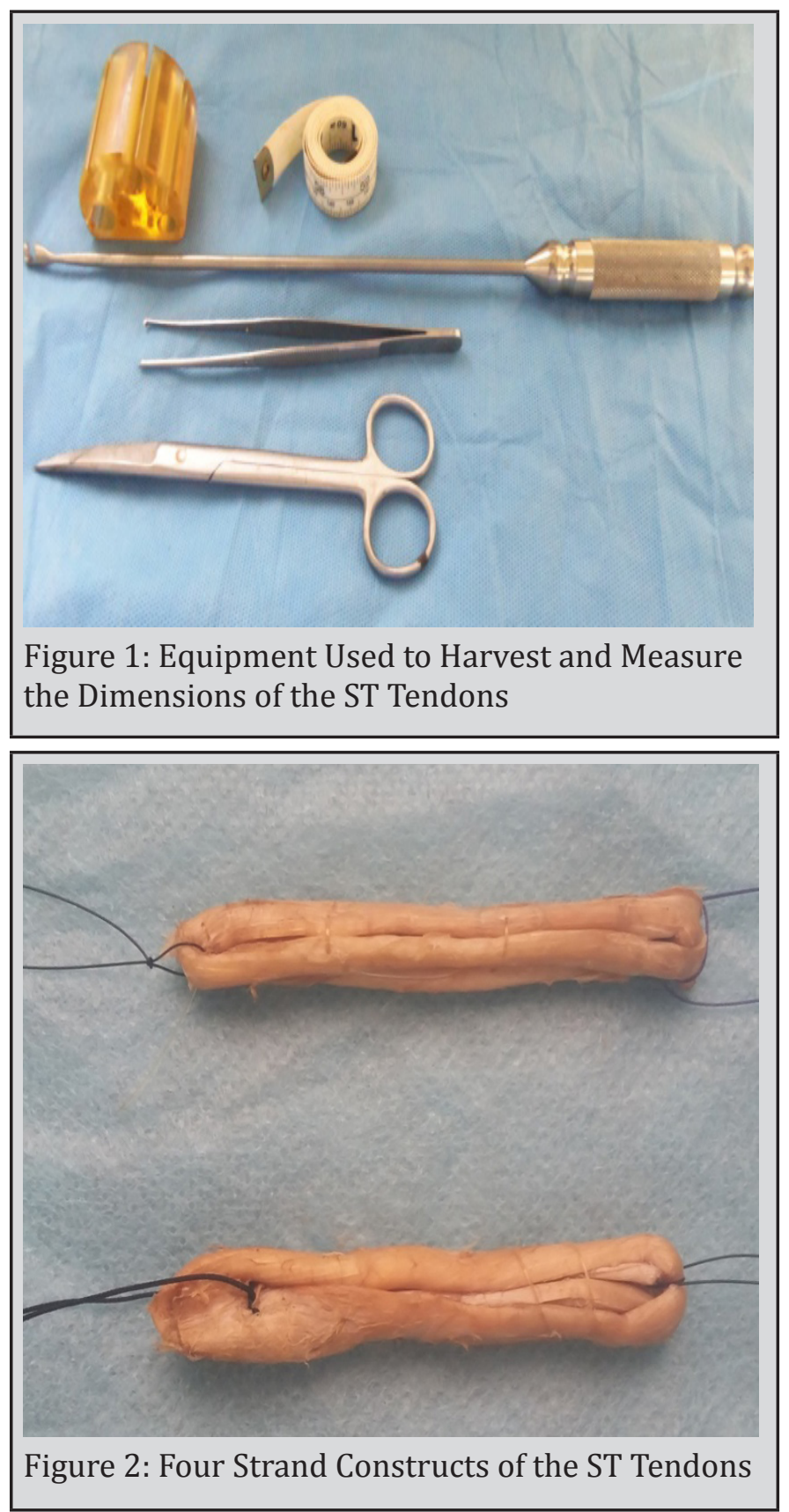

\section{Results}

The average ST tendon length was $29.80 \pm 3.59 \mathrm{~cm}$ and $67.5 \%$ of all tendons had a length $\geq 28.0 \mathrm{~cm}$. The left ST tendons were significantly longer than the right ST tendons ( $\mathrm{p}$ value 0.02 ). The four strand construct diameter was $7.849 \pm 0.658 \mathrm{~mm}$ and $56 \%$ of all tendons had a thickness $\geq 8.00 \mathrm{~mm}$. Considering tendon adequacy to be the presence of both sufficient ST tendon length and four strand construct diameter, $51 \%$ of all tendons were adequate for solitary use (Table 1 and 2).

Table 1: ST Tendon Graft Dimensions
\begin{tabular}{|l|l|l|l||}
\hline & Average & Right & Left \\
\hline ST tendon & 29.800 & 29.642 & 29.950 \\
length $(\mathrm{cm})$ & $( \pm 3.59)$ & $( \pm 3.699)$ & $( \pm 3.489)$ \\
\hline Four strand & 7.849 & 7.833 & 7.865 \\
diameter $(\mathrm{mm})$ & $( \pm 0.658)$ & $( \pm 0.598)$ & $( \pm 0.619)$ \\
\hline
\end{tabular}

\begin{tabular}{|l|l|l|}
\hline \multicolumn{3}{l}{ Table 2: Adequacy for Solitary Use } \\
\begin{tabular}{|l|l|l|}
\hline Dimension & Right & Left \\
\hline $\begin{array}{l}\text { ST tendon length } \\
\geq 28 \mathrm{~cm}\end{array}$ & $25 / 40(62.5 \%)$ & $29 / 40(72.5 \%)$ \\
\hline $\begin{array}{l}\text { Four strand } \\
\text { diameter } \geq 8 \mathrm{~mm}\end{array}$ & $23 / 40(57.5 \%)$ & $22 / 40(55.0 \%)$ \\
\hline $\begin{array}{l}\text { Length } \geq 28 \mathrm{~cm} \mathrm{\&} \\
\text { diameter } \geq 8 \mathrm{~mm}\end{array}$ & $20 / 40(50.0 \%)$ & $21 / 40(52.5 \%)$ \\
\hline
\end{tabular}
\end{tabular}

Table 3: Comparison on ST Graft for ACL Reconstruction

\begin{tabular}{|llll|}
\hline & Sample & $\begin{array}{l}\text { Length } \\
\text { (cm) }\end{array}$ & $\begin{array}{l}\text { Diameter } \\
(\mathrm{mm})\end{array}$ \\
$\begin{array}{l}\text { Challa and } \\
\text { Satyaprasad, } \\
\text { 2013 }\end{array}$ & 41 & 24.39 & 10.26 \\
$\begin{array}{l}\text { Pichler et., al } \\
\text { 2008 }\end{array}$ & 45 & 26.37 & 7.62 \\
$\begin{array}{l}\text { Xie et al., } \\
\text { 2012 }\end{array}$ & 235 & 27.90 & 11.90 \\
$\begin{array}{l}\text { Stergios et } \\
\text { al., 2012 }\end{array}$ & 61 & 29.39 & 7.30 \\
$\begin{array}{l}\text { Current study, } \\
\text { 2016 }\end{array}$ & & 29.80 & 7.85 \\
\hline
\end{tabular}




\section{Discussion}

The average length of the ST tendon in this study was $29.80 \mathrm{~cm}$ and over $67 \%$ of our population had a ST tendon whose length was at least $28 \mathrm{~cm}$. When compared to reports from Chinese, Austrian and Indian populations, our sample population had a longer ST tendon (18-20). Greeks, on the other hand, are reported to have longer tendons than observed in our sample population as summarized in table 3 (9). A search of literature at the time of conducting this study did not yield any publications on the ST tendon within the sub-Saharan region. Studies from Caucasian subpopulations generally report lower values for ST tendon length compared to reports among Greeks and the present study. Even though racial differences in height are a common explanation for this observation, it may not be entirely true to state that the short stature of the Caucasians ultimately results in shorter ST tendons. This is because correlations between ST tendon length and body height, in studies done among Caucasians, are generally weak (18). We hypothesized that ST tendon graft lengths may be a function of the muscle morphology. It has been reported that athletes of Kenyan descent have longer tendons in their triceps surae compared to their Caucasian counterparts (21). This suggests the existence of genetic differences in muscle morphology. It is therefore plausible that Kenyans may have longer tendinous portions of the ST muscle tendon unit resulting in longer tendon grafts. A four strand construct is a standard ST tendon graft design that provides adequate strength and thickness as an ACL replacement. The average four strand construct diameter obtained in our study was 7.849 $\mathrm{mm}$ and is comparable to those reported amongst Greeks and Austrians. However, thicker grafts have been reported among Indians and Chinese who are reported to have inadequate ST tendon graft lengths for solitary use (Table 3). An earlier report on the use of a solitary ST tendon to reconstruct the ACL proposed a minimum four strand thickness of $7 \mathrm{~mm}$, which is roughly the lower limit of the ACL diameter $(8,9)$. However, a $13.6 \%$ risk of revision has been reported in patients with graft diameters $\leq 7 \mathrm{~mm}$ compared to a $1.7 \%$ risk for patients with diameters $>8 \mathrm{~mm}$ (14). In our sample, $14 \%$ had a strand construct diameter less than $7 \mathrm{~mm}$ while $52 \%$ met the $8 \mathrm{~mm}$ cutoff. This represents a good proportion of our sample that would have a ST tendon graft adequate for solitary use.

With a good proportion of our sample population having an adequate ST tendon, it is encouraged that a solitary ST tendon be used for ACL reconstruction. Preoperative estimation of hamstring graft dimensions would help identify patients whose ST tendon graft may not be adequate and would need a $G$ tendon graft as well. However, it still remains difficult to determine, pre-operatively, the dimensions and adequacy of hamstring tendon grafts $(5,15,18)$. In addition, a literature search up until when this study was conducted did not yield any information as to which tendon is harvested first in the procedures leading to the reconstruction the ACL. This knowledge would help avoid unnecessary harvesting of G tendon if ST tendon is harvested first and found to be adequate. We suggest that ST be harvested first in all patients undergoing reconstruction and the need to harvest G tendon be determined after dimensions of the ST tendon graft have been established.

\section{Conclusion}

The use of semitendinosus as a solitary graft in ACL reconstruction may be feasible among Kenyans as a majority had adequate dimensions. We propose that the semitendinosus tendon be harvested first during reconstruction and its adequacy determined prior to harvesting the gracilis tendon.

\section{References}

1. Wasserstein D, Sheth U, Cabrera A, et al. A Systematic Review of Failed Anterior Cruciate Ligament Reconstruction with Autograft Compared with Allograft in Young Patients. Sports Health Multidiscip Approach. 2015; 7(3):207-16.

2. Macaulay AA, Perfetti DC, Levine WN. Anterior Cruciate Ligament Graft Choices. Sports Health. 2012; 4(1):63-8.

3. Shaerf DA, Pastides PS, Sarraf KM, et al. Anterior Cruciate Ligament Reconstruction Best Practice: A Review of Graft Choice. World J Orthop. 2014; 5(1):23-9.

4. Barenius B, Webster WK, McClelland J, et al. Hamstring Tendon Anterior Cruciate Ligament Reconstruction: Does Gracilis Tendon Harvest Matter? Int Orthop. 2013; 37(2):207-12.

5. Conte EJ, Hyatt AE, Gatt Jr. CJ, et al. Hamstring Autograft Size can be Predicted and is a Potential Risk Factor for Anterior Cruciate Ligament Reconstruction Failure. Arthrosc J Arthrosc Relat Surg. 2014; 30(7):882-90.

6. Gobbi A, Francisco R. Factors Affecting Return to Sports after Anterior Cruciate Ligament Reconstruction with Patellar Tendon and Hamstring Graft: A Prospective Clinical Investigation. Knee Surg Sports Traumatol Arthrosc. 2006; 14(10):1021-8.

7. Weiler A, Scheffler SU, Südkamp NP. Current Aspects of Anchoring Hamstring Tendon Transplants in Cruciate Ligament Surgery. Chir Z Für Alle Geb Oper Medizen. 2000; 71(9): 1034-44. 
8. Gobbi A, Domzalski M, Pascual J, et al. Hamstring Anterior Cruciate Ligament Reconstruction: Is it Necessary to Sacrifice The Gracilis? Arthrosc J Arthrosc Relat Surg. 2005; 21(3):275-80.

9. Stergios PG, Georgios KA, Konstantinos N, et al. Adequacy of Semitendinosus Tendon Alone for Anterior Cruciate Ligament Reconstruction Graft and Prediction of Hamstring Graft Size by Evaluating Simple Anthropometric Parameters. Anat Res Int. 2012; 2012:1-8.

10. Królikowska A, Czamara A, Kentel M. Does Gracilis Tendon Harvest During ACL Reconstruction With A Hamstring Autograft Affect Torque Of Muscles Responsible For Shin Rotation? Med Sci Monit Int Med J Exp Clin Res. 2015; 21:2084-93.

11. Thomas AC, Villwock M, Wojtys EM, et al. Lower Extremity Muscle Strength after Anterior Cruciate Ligament Injury and Reconstruction. J Athl Train. 2013; 48(5):610-20.

12. Battaglia T, Miller MD. Strength and Regrowth Of Hamstring Tendons after Autograft Anterior Cruciate Ligament Reconstruction. Tech Orthop. 2005; 20(3):334-9.

13. Sasahara J, Takao M, Miyamoto W, et al. Partial Harvesting Technique in Anterior Cruciate Ligament Reconstruction with Autologous Semitendinosus Tendon to Prevent a Postoperative Decrease in Deep Knee Flexion Torque. The Knee. 2014; 21(5):936-43.

14. Magnussen RA, Lawrence JTR, West RL, et al. Graft Size and Patient Age are Predictors of Early Revision after Anterior Cruciate Ligament Reconstruction with Hamstring Autograft. Arthrosc J Arthrosc Relat Surg. 2012; 28(4):526-31.
15. Erquicia JI, Gelber PE, Doreste JL, et al. How to Improve the Prediction of Quadrupled Semitendinosus and Gracilis Autograft Sizes with Magnetic Resonance Imaging and Ultrasonography. Am J Sports Med. 2013; 41(8):1857-63.

16. Charalambous CP, Kwaees TA. Anatomical Considerations in Hamstring Tendon Harvesting for Anterior Cruciate Ligament Reconstruction. Muscles Ligaments Tendons J. 2013; 2(4):253-7.

17. Limitlaohaphan C, Kijkunasatian C, Saitongdee P. Length of Semitendinosus and Gracilis Tendons and the Relationship of Graft Length and Leg Length. J Med Assoc Thail Chotmaihet Thangphaet. 2009; 92 Suppl 6:S200-203.

18. Xie G, Huangfu X, Zhao J. Prediction of the Graft Size of 4-Stranded Semitendinosus Tendon and 4-Stranded Gracilis Tendon for Anterior Cruciate Ligament Reconstruction A Chinese Han Patient Study. Am J Sports Med. 2012; 40(5):1161-6.

19. Challa S, Satyaprasad J. Hamstring Graft Size and Anthropometry in South Indian Population. J Clin Orthop Trauma. 2013; 4(3):135-8.

20. PichlerW, Tesch NP, Schwantzer G, etal. Differences in Length and Cross-Section of Semitendinosus and Gracilis Tendons and Their Effect on Anterior Cruciate Ligament Reconstruction: A Cadaver Study. J Bone Joint Surg Br. 2008; 90-B(4):516-9.

21. Sano K, Ishikawa M, Nobue A, et al. MuscleTendon Interaction and EMG Profiles of World Class Endurance Runners During Hopping. Eur J Appl Physiol. 2013; 113(6):1395-403. 